\title{
NOTE
}

\section{INTERNATIONAL BOTANICAL CONGRESS} STOCKHOLM, 1940

Motions dealing with Nomenclature for consideration by the Seventh International Botanical Congress, Stockholm, 1940, should be sent before July 1, 1939, to the Rapporteur général, Dr. T. A. Sprague, The Herbarium, Royal Botanic Gardens, Kew, Surrey, England.

Motions must be presented in the form of additional articles or amendments to the International Rules. They should be drafted as briefly as possible. At least 100 printed copies must be presented.

Section for Taxonomy and Nomenclature RECORDER :

Dr. J. A. Nannfeldt 


\section{$2 \mathrm{BHL}$ Biodiversity Heritage Library}

Nannfeldt, J A. 1939. "Note: VII. International Botanical Congress, Stockholm, 1940." Journal of the Arnold Arboretum 20(1), 138-138.

https://doi.org/10.5962/p.185404.

View This Item Online: $\underline{\text { https://www.biodiversitylibrary.org/item/33596 }}$

DOI: https://doi.org/10.5962/p.185404

Permalink: https://www.biodiversitylibrary.org/partpdf/185404

\section{Holding Institution}

Missouri Botanical Garden, Peter H. Raven Library

\section{Sponsored by}

Missouri Botanical Garden

\section{Copyright \& Reuse}

Copyright Status: In copyright. Digitized with the permission of the rights holder.

Rights Holder: Arnold Arboretum of Harvard University

License: http://creativecommons.org/licenses/by-nc-sa/3.0/

Rights: https://biodiversitylibrary.org/permissions

This document was created from content at the Biodiversity Heritage Library, the world's largest open access digital library for biodiversity literature and archives. Visit BHL at https://www.biodiversitylibrary.org. 